\title{
MiR-589-5p is a potential prognostic marker of hepatocellular carcinoma and regulates tumor cell growth by targeting MIG-6
}

\author{
M. XU, Y. WANG, H. T. HE, Q. YANG* \\ Department of Pathogenobiology, College of Basic Medical Sciences, Jilin University, Changchun, China \\ ${ }^{*}$ Correspondence: yangq@jlu.edu.cn
}

Received November 25, 2017 / Accepted March 15, 2018

\begin{abstract}
MicroRNAs (miRNAs) are small noncoding RNAs approximately with 22 nucleotides. Accumulating evidence indicates that they are involved in carcinogenesis and tumor progression and recent investigations have also reported that several microRNAs can act as biomarkers in cancer diagnosis and prognosis. MicroRNA-589-5p (miR-589-5p) is a less studied microRNA, and herein we examined its roles in hepatocellular carcinoma (HCC). We analyzed miR-589-5p expression in HCC tissues by sequencing data and proved its expression in liver cancer cell lines by quantitative real-time PCR (qRT-PCR). We then studied its effect on liver cancer cell growth by MTT assay, colony formation and flow cytometry, and identified its target gene by luciferase reporter assay. We found that miR-589-5p was commonly over-expressed in HCC specimens. High expression of miR-589-5p was a risk factor for HCC patients (Hazard ratio [HR]=1.434; 95\% confidence intervals $[\mathrm{CI}]=1.006-2.044 ; \mathrm{p}=0.046$ ). We also found miR-589-5p had higher expression in the HepG2 and HuH-7 hepatocarcinoma cell lines than in the normal Lo-2 hepatocyte. We established that suppression of miR-589-5p inhibited cell proliferation and cell cycle progression by loss-of-function studies. Furthermore, we found mitogen-inducible gene 6 (MIG-6) is a target for miR-589-5p. Our study demonstrated that miR-589-5p facilitated the growth of liver cancer cells by targeting MIG-6 and that it could be a prognosis biomarker for HCC. Suppression of miR-589-5p may be a feasible approach for inhibiting HCC progress.
\end{abstract}

Key words: hepatocellular carcinoma, microRNA-589-5p, prognosis, MIG-6

Liver cancer is one of the most common global digestive system cancers [1]. An estimated 40,710 new cases of liver and intrahepatic bile duct cancer were expected in the US in 2017 [2]. Hepatocellular carcinoma (HCC) is the most prevalent subtype of liver cancer, accounting for approximately $85-90 \%$ of all patients [3] and patient prognosis has significantly improved in the past decades with technological advances in early detection and intervention. However, prognosis still remains poor for those with advanced stage HCC; even for those receiving standard care by surgical resection and liver transplantation $[4,5]$. It is therefore imperative to identify novel biomarkers at the molecular level and further elucidate the HCC molecular mechanisms.

MicroRNAs (miRNA) are a family of small non-coding RNA molecules that down-regulate the expression of their target genes at the post-transcriptional level. These RNAs are 19-25 nucleotides long and can bind to the complementary sequence of their target mRNAs' 3'untranslated region (3'UTR) [6]. MicroRNAs are considered to be involved in many important biological processes including cell prolif- eration, migration, invasion and apoptosis [7]. They are also widely reported to play a vital role in regulating tumor biological behavior; with dysregulated miRNAs expression found in various tumor types [8, 9]. MicroRNA-589-5p (miR-589-5p) located on chromosome 7 (7p22.1) is a less studied miRNA and its role in cancer is poorly understood.

Herein, we found that miR-589-5p was up-regulated in HCC tissues and cells, and higher expression of miR-589-5p in HCC samples was related to shorter overall patient survival. We identified that MIG-6 was a miR-589-5p target gene, and that miR-589-5p suppression increased MIG-6 expression and inhibited tumor cell growth. Our research revealed that miR-589-5p promotes the growth of HCC cells by targeting MIG-6 and therefore suggests miR-589-5p as a novel HCC prognosis biomarker.

\section{Patients and methods}

Patient information and miRNA sequencing data. The miRNA expression data and corresponding clinical infor- 
mation of the patients with liver cancer were collected from The Cancer Genome Atlas (TCGA) data portal. MiRNA and mRNA sequencing was performed by the Illumina HiSeq platforms (Illumina Inc, San diego, Ca, USA) and we downloaded standardized level 3 data from the Standardized Data Browser (http://bioinformatics.mdanderson.org/ TCGA/databrowser/). Clinical information and demographic characteristics of patients enrolled in this study are summarized in Table 1.

Cell lines and culture. The HepG2 and HuH-7 human HCC cell lines and human embryonic kidney cell line HEK-293T were obtained from the Cell Bank of the Chinese Academy of Sciences (Shanghai, China). These cells were stored in liquid nitrogen and cultured in Dulbecco's Modified Eagle Medium (DMEM) medium (Gibco, USA) supplemented with penicillin $(100 \mathrm{IU} / \mathrm{mL}$ ) and $10 \%$ FBS (fetal bovine serum) at $37^{\circ} \mathrm{C}$ and $5 \% \mathrm{CO}_{2}$.

RNA isolation and quantitative real-time PCR (qRT-PCR). Total RNAs were isolated from cells by Trizol (TaKaRa, China) according to protocol and RNA concen-

Table 1. Clinical characteristics of HCC patients.

\begin{tabular}{|c|c|c|c|}
\hline Characteristics & $\begin{array}{c}\text { Low miR-589-5p } \\
\text { expression group } \\
\quad(n=174)\end{array}$ & $\begin{array}{l}\text { High miR-589-5p } \\
\text { expression group } \\
\quad(n=174)\end{array}$ & p-value ${ }^{a}$ \\
\hline Age & $59.8 \pm 13.7$ & $59.7 \pm 12.9$ & 0.967 \\
\hline \multicolumn{4}{|l|}{ Gender } \\
\hline Male & 118 & 120 & 0.818 \\
\hline Female & 56 & 54 & \\
\hline AJCC stage & & & 0.639 \\
\hline I & 87 & 77 & \\
\hline II & 40 & 39 & \\
\hline III & 34 & 45 & \\
\hline IV & 2 & 1 & \\
\hline NA & 11 & 12 & \\
\hline Tumor size & & & 0.713 \\
\hline $\mathrm{T} 1$ & 92 & 81 & \\
\hline $\mathrm{T} 2$ & 41 & 44 & \\
\hline $\mathrm{T} 3$ & 34 & 41 & \\
\hline $\mathrm{T} 4$ & 5 & 7 & \\
\hline $\mathrm{TX}$ & 2 & 1 & \\
\hline \multicolumn{4}{|l|}{ Lymph node } \\
\hline No & 114 & 127 & 0.097 \\
\hline N1 & 3 & 0 & \\
\hline NX & 57 & 47 & \\
\hline Metastasis status & & & 0.031 \\
\hline M0 & 114 & 136 & \\
\hline M1 & 2 & 1 & \\
\hline MX & 58 & 37 & \\
\hline Vital status & & & 0.074 \\
\hline Live & 119 & 103 & \\
\hline Dead & 55 & 71 & \\
\hline
\end{tabular}

AJCC: American Joint Committee on Cancer; MX: metastasis status un-

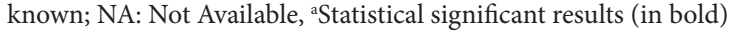

tration was measured by BioSpectrometer (Eppendorf, Germany). cDNAs were synthesized by TransScript RT reagent Kit (TransGen, China) and QRT-PCR was performed with FastStart Universal SYBR Green Master (ROX) (Roche,USA) and CFX96 Real-Time system (Bio-Rad, USA). The U6 small nuclear RNA was used as control for normalization in detected miR-589-5p expression and $\beta$-actin was the control for normalization in detected MIG-6 expression. $\beta$-actin primers were 5'-CTGGAACGGTGAAGGTGACA-3' and 5'-AAGGGACTTCCTGTAACAATGCA-3'; MIG-6 primers were 5'-C TACTGGAGCAGTCGCAGTG3'and 5'-CCTCTTCATGTGGTCCCAAG-3'. Each experiment was repeated at least three times and data was analyzed by the $2^{-\Delta \Delta} \mathrm{CT}$ method.

Transient transfection. The MiR-589-5p inhibitors, miR-589-5p inhibitors negative control (NC), miR-589-5p mimics and miR-589-5p mimic negative control (NC) were synthesized by Ribobio (Guangzhou, China). SiRNAs for MIG-6 were purchased from Gene Pharma (Shanghai, China) and oligonucleotides were transfected into cells using Lipofectamine 2000 (Invitrogen, USA) according to the manufacturer's guidelines.

Cell growth, colony formation and cell cycle assays. For MTT assay, HCC cells transfected with oligonucleotides were seeded into 96 -well plates at $5 \times 10^{3}$ cells/well density, and absorbance at $490 \mathrm{~nm}$ was measured on days $1,2,3,4$ and 5. 500 HCC cells were then incubated in 6-well plates at $37^{\circ} \mathrm{C}$ and $5 \% \mathrm{CO} 2$ for cell colony formation, and these were transfected with miR-589-5p inhibitors or NC. After two weeks, the cells were stained with crystal violet $(0.2 \%)$ for 30 minutes and colony numbers were counted. Cell cycle analysis was performed according to the protocol of the standard propidium iodide (PI) method. After $24 \mathrm{~h}$ transfection, the cells were firstly trypsinized and washed with cold PBS and then fixed in 70\% ethanol for 24 hours and stained with PI for 30 minutes. Finally, the cells were analyzed by Accuri C6 flow cytometer (BD, USA). Each experiment was performed at least in triplicate.

Protein extraction and Western blot. Total proteins were extracted from cultured cells using cell lysis buffer. The protein samples were then loaded onto $10 \%$ sodium dodecyl sulfate polyacrylamide gel electrophoresis (SDS-PAGE). The membranes were blocked with $5 \%$ non-fat milk in Tris-buffered saline and incubated with a specific primary antibody and a secondary antibody. Protein expression was detected by enhanced chemiluminescence (ECL) kit, with $\beta$-actin as the loading control. The antibodies for MIG-6 (D261911) were purchased from Sangon Biotech and $\beta$-actin antibody and secondary antibodies were purchased from Beyotime.

Luciferase reporter assay. The pmir-GLO Dual-Luciferase miRNA Target Expression Vector for miR-589-5p target MIG-6 3'UTR was constructed as wtMIG6-3'-UTR containing the wild-type 3'UTR of MIG-6, and as mtMIG63'-UTR containing the mutant 3'UTR of MIG-6. The wtMIG6- 
3'-UTR vector and mtMIG6-3'-UTR vector containing Firefly luciferase and Renilla luciferase. HEK-293T cells were seeded into 96-well plates, and each was co-transfected with the wtMIG6-3'-UTR vector and miR-589-5p mimics or NC mimics, or co-transfected with the mtMIG6-3'-UTR vector and miR-589-5p mimics or NC mimics by Lipofectamine 2000 (Invitrogen) according to the manufacturer's protocol. The luciferase activity was measured by the Dual Luciferase Assay system (Promega) 48 hours after transfection and Renilla luciferase activity was normalized to Firefly luciferase activity (Renilla LUC/ Firefly LUC). Each experiment was performed at least three times.

Statistical analysis. All statistical analyses were performed using SPSS 18.0 (SPSS Inc., Chicago, USA) and GraphPad Prism 6 (GraphPad Software Inc., CA, USA) software. The Chi-square test analyzed the different distribution of clinical variables, and differences in the miR-589-5p and MIG-6 expression levels between HCC and corresponding normal tissues were analyzed by paired student's t-test. The Univariate Cox proportional hazards regression model then analyzed the potential factors associated with survival. Overall survival was estimated by the Kaplan-Meier method. For in vitro experiments, the t-test or analysis of variance (ANOVA) evaluated the difference between the two groups. All p-values were two sided, with $\mathrm{p}<0.05$ statistically significant. All data is presented as the mean \pm standard deviation (SD) from at least three independent replicates.

\section{Results}

MiR-589-5p was up-regulated in HCC tissues and this correlated with HCC patient survival. Sequencing data downloaded from the TCGA database showed altered expression of miRNAs in HCC tissues compared to corresponding non-tumor tissues (Figure 1A). We found that the mean miR-589-5p expression level (marked with green
A

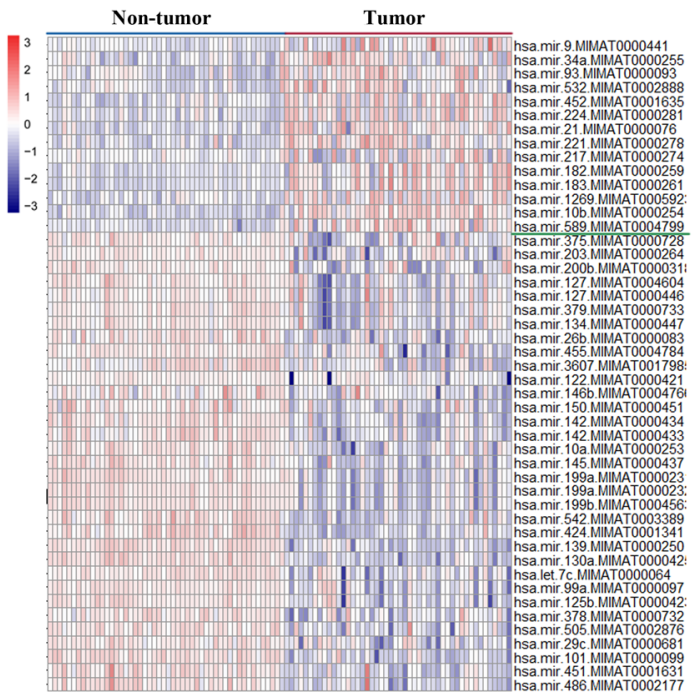

B

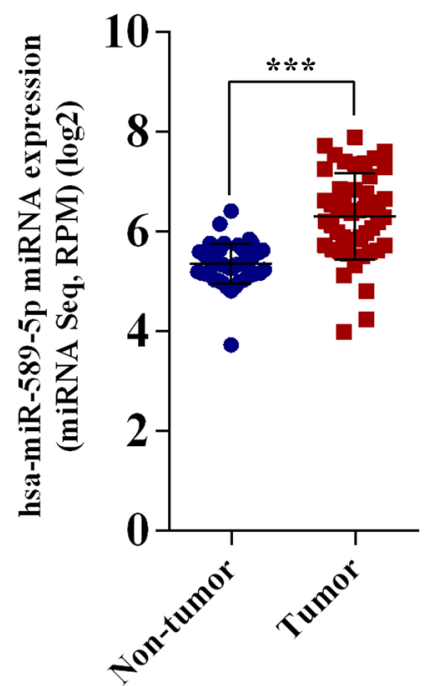

C

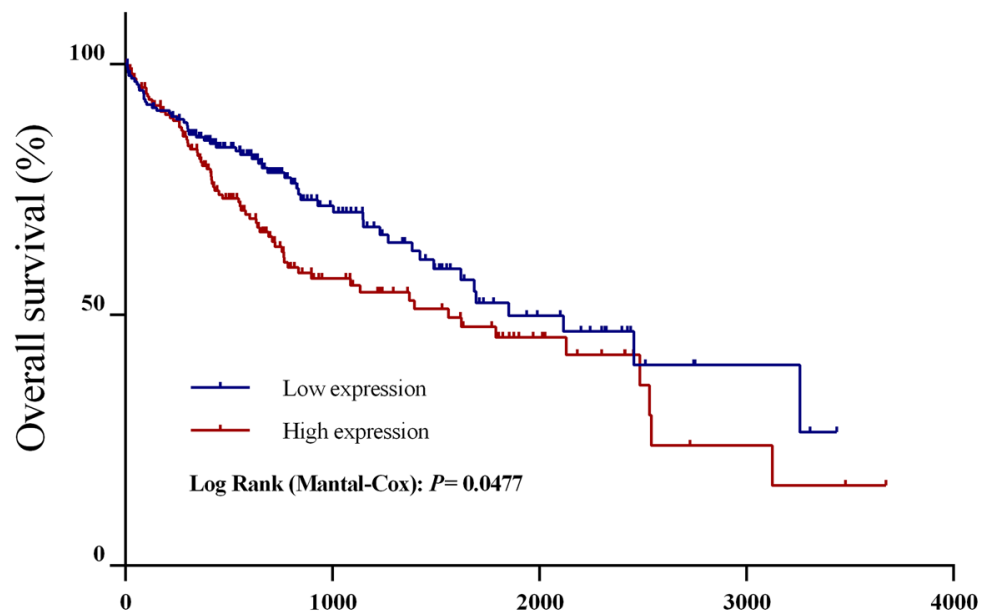

Figure 1. MiR-589-5p is highly expressed in HCC tissues and its expression in cancer tissues is related to patient overall survival. A) Hierarchical cluster heat map of differentially expressed miRNAs in HCC and corresponding normal tissues generated from miRNA sequencing data from the TCGA database. Red in the heat map denotes up-regulation; blue denotes down-regulation. The green arrow indicates miR-5895 p. B) Up-regulation of miR-589-5p in HCC tissues. ${ }^{* * *} \mathrm{p}<0.001$. C) HCC patients whose tumors expressed elevated levels of miR-589-5p had poor survival compared to those with low levels, at $\mathrm{p}=\mathbf{0 . 0 4 7 7}$. 
A

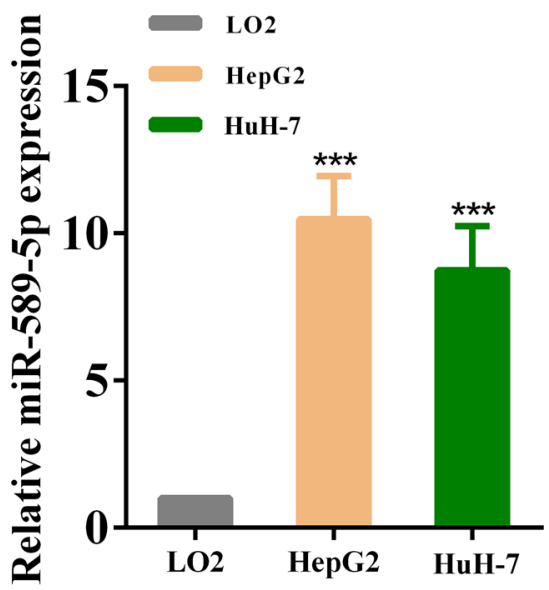

C

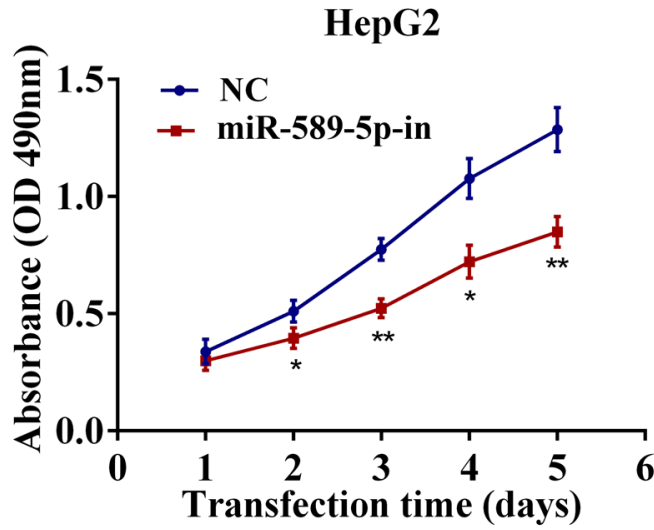

D

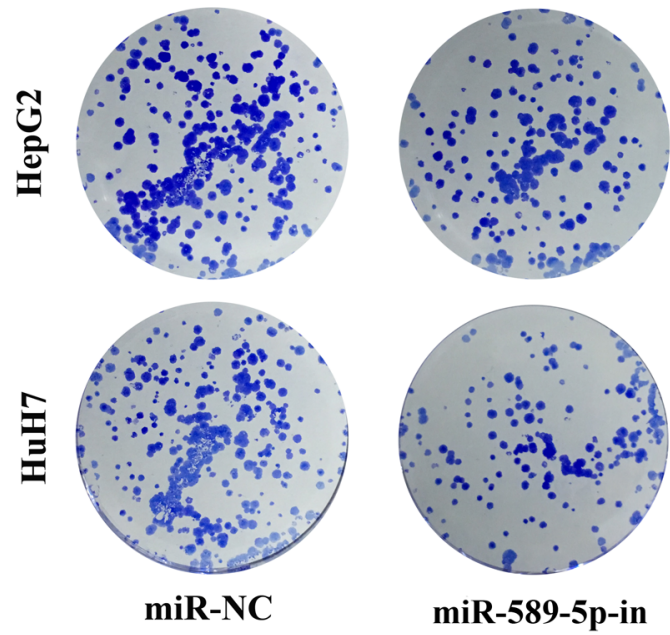

B
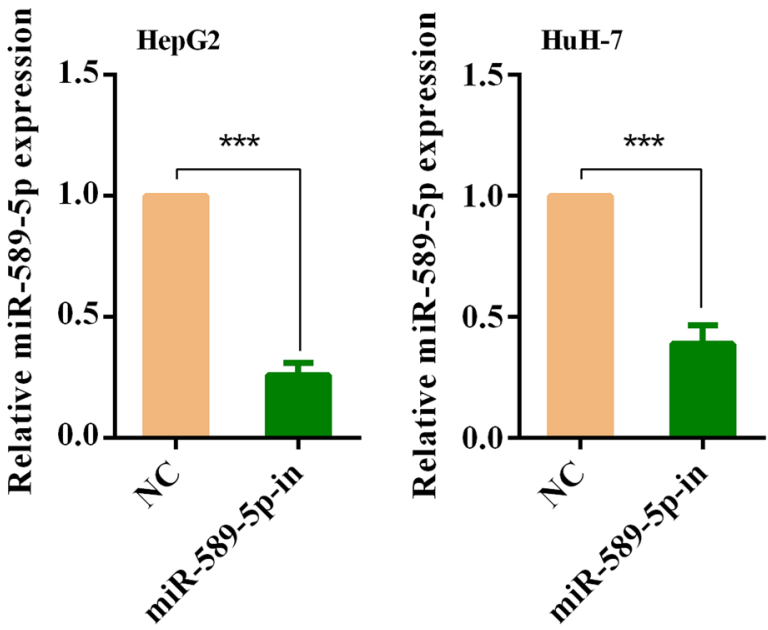

HuH-7

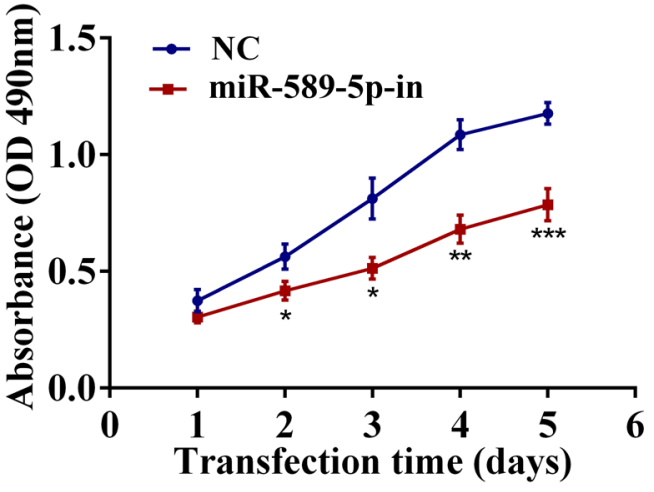

HepG2

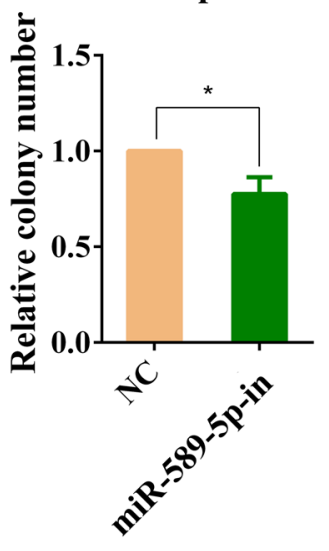

НuH-7

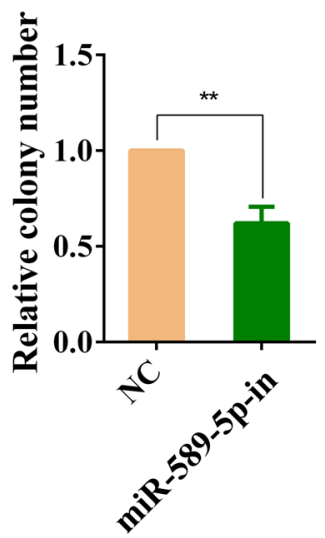

Figure 2. MiR-589-5p affects viability and colony formation of HepG2 and HuH-7 cells. A) Up-regulation of miR-589-5p in HepG2 and HuH-7 cells. ${ }_{* * *} \mathbf{p}<0.001$. B) QRT-PCR analysis of miR-589-5p expression in HepG2 and HuH-7 cells transfected with miR-589-5p inhibitors. ${ }^{* * *} \mathbf{p}<0.001$. C) Cell viability assay. HepG2 and HuH-7 cells transfected with miR-589-5p inhibitors were subjected to MTT assay $24 \mathrm{~h}$ after transfection. ${ }^{*} \mathrm{p}<0.05,{ }^{* *} \mathrm{p}<0.01$ and ${ }^{\star * *} \mathbf{p}<0.001$. D) Effects of miR-589-5p on colony formation of HCC cells. HepG2 and HuH-7 cells transfected with miR-589-5p inhibitors were seeded onto 6-well plates. The number of colonies was counted on the 14th day after seeding providing representative micrographs (left) and relative quantification analysis of colonies (right). ${ }^{*} \mathrm{p}<0.05$ and ${ }^{* *} \mathrm{p}<0.01$. 
underline in Figure 1A) was higher in the tumor tissues than in matched non-tumor tissues (Figure 1B). High miR-589-5p expression was identified as a risk factor for HCC patients by the univariate Cox proportional hazards regression analysis, (Table 2) and survival analysis using the KaplanMeier method also showed that higher miR-589-5p levels were associated with shorter HCC patient overall survival (Figure 1C). These results indicate that deregulated expression of miR-589-5p is a common event in HCC tissues, and that miR-589-5p should therefore be a novel prognostic biomarker for HCC.

MiR-589-5p was up-regulated in HCC cell lines. We next assessed miR-589-5p expression in HCC cells by qRT-PCR, and this established that miR-589-5p expression was significantly up-regulated in the HepG2 and $\mathrm{HuH}-7$ HCC cell lines compared to the normal human hepatic Lo-2 cells (Figure 2A).

Down-regulation of miR-589-5p inhibited HCC cell growth and colony formation. Because of the general increase of miR-589-5p expression in HCC tissues and cells, we hypothesized that a high level of miR-589-5p could be involved in HCC cell growth and therefore we transfected miR-589-5p inhibitors into the HepG2 and $\mathrm{HuH}-7$ cells. Figure 2B indicated that this transfection significantly suppressed miR-589-5p expression, and MTT and colony formation assays established that cell growth (Figure 2C) and colony formation (Figure 2D) were significantly inhibited in HepG2 and $\mathrm{HuH}-7$ cells transfected with miR-589-5p inhibitors.
Table 2. Univariate Cox proportional hazards regression analysis of factors associated with overall survival.

\begin{tabular}{lccc}
\hline & & HR $(\mathbf{9 5 \%}$ CI $)$ & p-value $^{\mathbf{a}}$ \\
\hline $\begin{array}{l}\text { MiR-589-5p } \\
\text { expression }\end{array}$ & $\begin{array}{c}\text { High vs. Low } \\
\text { expression }\end{array}$ & $1.434(1.006-2.044)$ & $\mathbf{0 . 0 4 6}$ \\
Gender & Female vs. Male & $1.163(0.809-1.672)$ & 0.416 \\
Age & $\geq 60$ vs. $<60$ & $1.284(0.897-1.838)$ & 0.172 \\
AJCC Stage & III + IV vs. I + II & $2.384(1.635-3.456)$ & $<\mathbf{0 . 0 0 1}$ \\
Tumor size & T3+T4 vs. T1+T2 & $2.505(1.750-3.586)$ & $<\mathbf{0 . 0 0 1}$ \\
Lymph node & N1 vs. N0 & $2.003(0.491-8.174)$ & 0.330 \\
Metastasis status & M1 vs. M0 & $3.933(1.236-12.511)$ & $\mathbf{0 . 0 2 0}$ \\
\hline
\end{tabular}

AJCC American Joint Committee on Cancer; HR hazard ratio, CI confi-

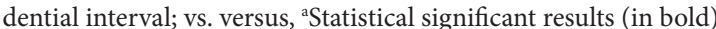

Down-regulation of miR-589-5p inhibited HCC cell cycle progression. We employed flow cytometry to determine miR-589-5p's role in the cell cycle and to elucidate the mechanism by which miR-589-5p suppression inhibited HCC cell growth. Figure 3 shows that miR-589-5p suppression led to a decline in the proportion of both HepG2 and $\mathrm{HuH}-7$ cells in the $\mathrm{S}$ phase. This further suggests miR-589-5p has an important role in promoting HCC cell growth by regulating the cell cycle.

MIG-6 was a target gene of miR-589-5p. We then assessed the molecular mechanisms by which miR-589-5p modulates HCC cell growth by investigating the miR-589-5p target genes. We searched for putative miR-589-5p targets in
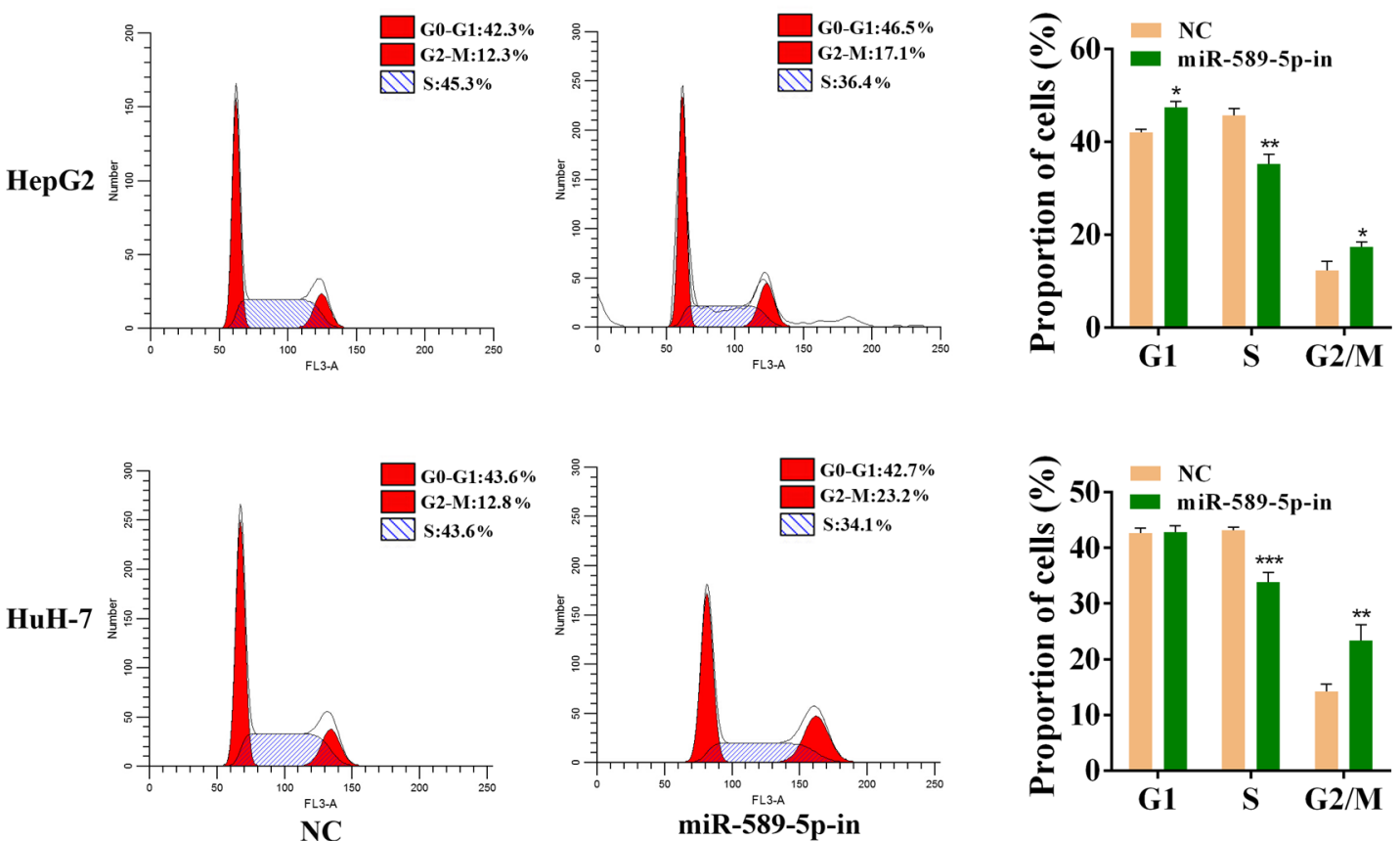

Figure 3. MiR-589-5p affects cell cycle progression of HepG2 and HuH-7 cells. Histograms depict the proportion of HepG2 and HuH-7 cells in G1, S, and G2/M phase after transfection with miR-589-5p inhibitors. ${ }^{*} \mathrm{p}<0.05,{ }^{* *} \mathrm{p}<0.01$ and ${ }^{\star * *} \mathrm{p}<0.001$. 
the following public bioinformatics tools: TargetScan [10], StarBase V2.0 [11] and miRDB [12]. MIG-6 was chosen from these theoretical targets for further analysis because it inhibits cell cycle progression and acts as a HCC tumor suppressor gene. We firstly analyzed the sequencing data downloaded from the TCGA database. Consistent with our speculation, the mean expression level of MIG-6 mRNA was lower in HCC tissues than in matched non-tumor tissues (Figure 4A). Then, to determine whether miR-589-5p regulated MIG-6 at the mRNA or protein level, we examined MIG-6 expression by qRT-PCR and Western blot after suppressing miR-589-5p.

Figures $4 \mathrm{~B}$ and $\mathrm{C}$ show that MIG-6 mRNA and proteins
A

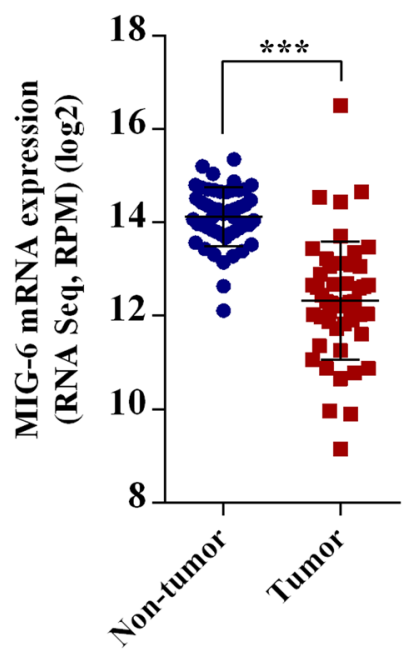

C

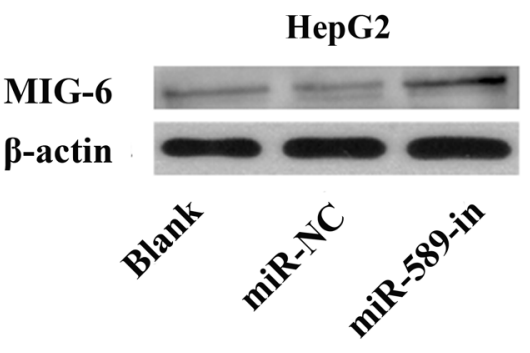

HuH-7

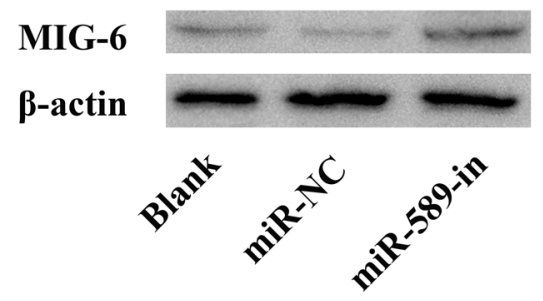

B
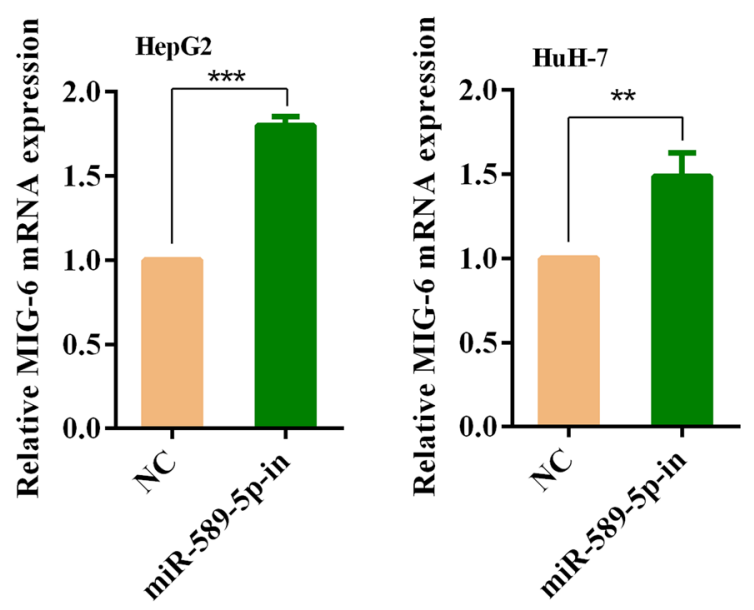

D

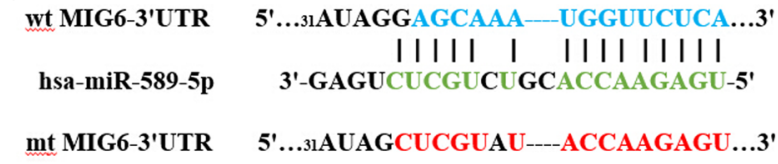

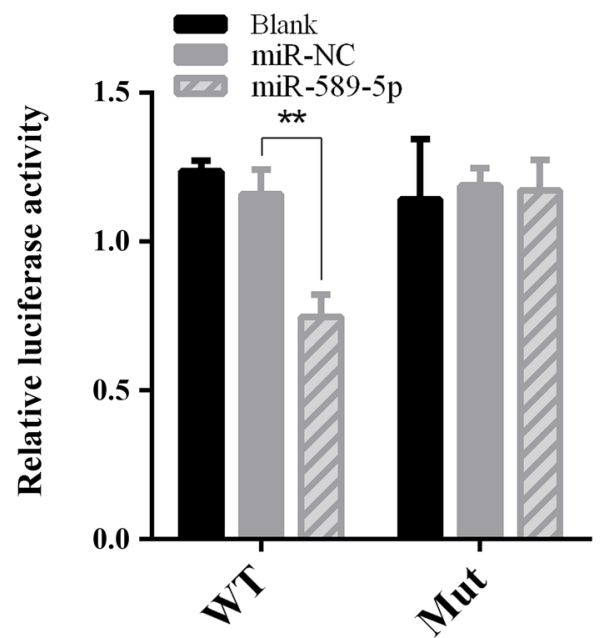

Figure 4. MiR-589-5p suppresses MIG-6 expression by directly targeting the MIG-6 3'-UTR. A) Down-regulation of MIG-6 in HCC tissues. ${ }^{* * *}<<0.001$. B) MIG-6 mRNA levels were detected by qRT-PCR in HepG2 and HuH-7 cells transfected with miR-589-5p inhibitors, respectively. ${ }^{* *}<0.01$ and ${ }_{* * *} \mathbf{p}<0.001$. C) Western Blot analysis of MIG-6 expression. MIG-6 protein levels were detected by Western blot in HepG2 and HuH-7 cells transfected with miR-589-5p inhibitors, respectively. D) Luciferase reporter assay. Predicted miR-589-5p target sequence (blue) in the 3'UTR of MIG-6 (wtMIG63'-UTR) and the position of mutated nucleotides (red) in the 3'-UTR of MIG-6 (mutMIG6-3'-UTR). A vector containing wild type MIG-6 3'-UTR or mutant MIG-6 3'UTR was co-transfected into HEK-293T cells together with indicated oligonucleotides. Luciferase activity ratio is presented as firefly luciferase value/renilla luciferase value. ${ }^{* *} \mathrm{p}<0.01$. 
levels were up-regulated in HepG2 and HuH-7 cells transfected with miR-589-5p inhibitors.

Two dual luciferase vectors containing either wildtype or mutated miR-589-5p targeting sequences were then constructed to identify the direct binding of miR-589-5p to MIG-6 (Figure 4D). Assay revealed decreased luciferase activity in HEK-293T cells transfected with the vector carrying wildtype miR-589-5p targeting sequences and miR-589-5p mimics, while luciferase activity in HEK-293T cells transfected with the vector carrying mutated miR-589-5p targeting sequences and miR-589-5p mimics remained unchanged. This combined data indicates that MIG- 6 is a direct target gene of miR-589-5p.

MiR-589-5p exerted its function by suppressing MIG-6 expression. HepG2 cells were co-transfected with miR-589-5p inhibitors and MIG-6 siRNA in order to determine if miR-589-5p exerts its function via MIG-6. MTT and cell colony assays indicated that cell growth inhibition induced by miR-589-5p knockdown was relieved by transfection with
MIG-6 siRNA (Figure 5A, 5B) and Flow Cytometry showed that the cell cycle arrest caused by miR-589-5p inhibition was also attenuated by MIG-6 silencing (Figure 5C).

\section{Discussion}

HCC is one of the most common cancers and a leading cause of mortality worldwide. Despite notable advances in diagnosis and treatment, the prognosis of patients with HCC has not improved significantly over the past years [13]. Therefore, it is urgent to elucidate the molecular mechanisms responsible for tumorigenesis and progression of HCC. Dysregulation of the oncogene and tumorsuppressor gene has been identified as a hallmark of cancer [14]. Many microRNAs are reported to play important roles in regulating gene expression in various tumors and miRNA expression profiling and function is becoming an important field in cancer research $[8,9,15]$. Further, miR-1, miR-21, miR-122, miR-221, miR-29, miR-500 and many
A

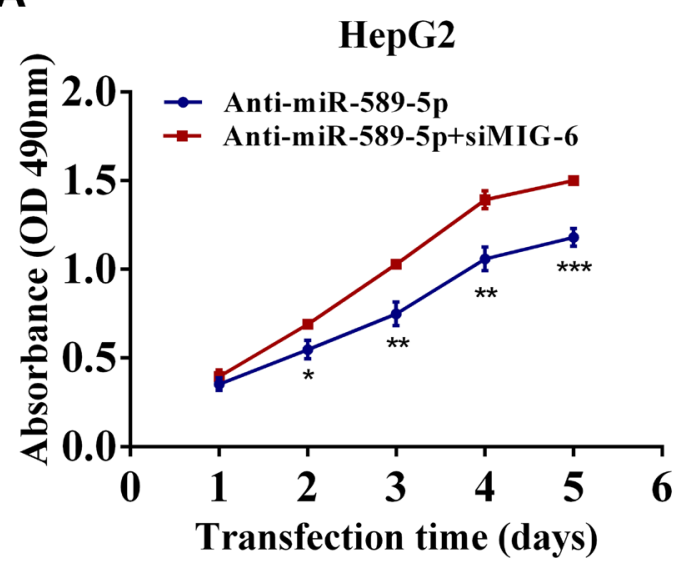

C

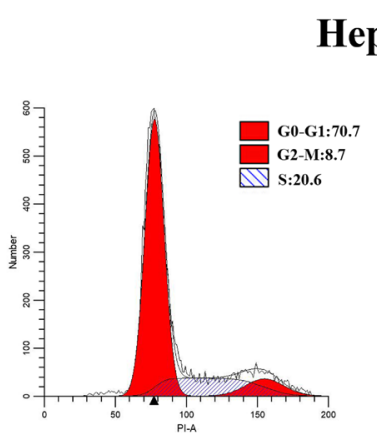

B
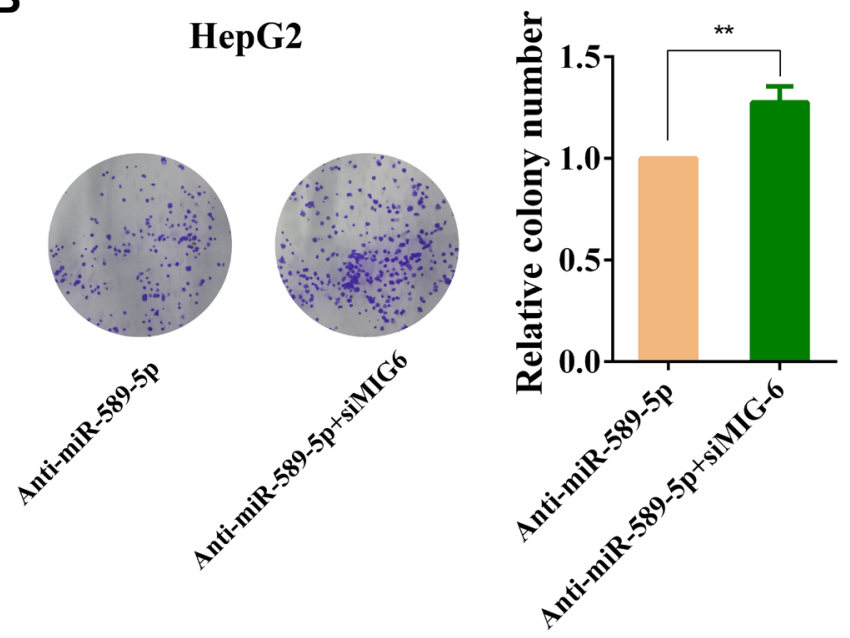

Figure 5. MIG-6 mediates the effects of miR-589-5p in HepG2 cells. A) MTT assay. Cell viability was assessed after MIG-6 siRNA was transfected into miR-589-5p inhibited HepG2 cells. ${ }^{*} p<0.05$, ${ }^{* *} p<0.01$ and ${ }^{* * *} p<0.001$. B) Colony formation assay. Transfection of MIG-6 siRNA in miR-589-5p inhibited HepG2 cells promoted cell colony formation. ${ }^{\star *} \mathrm{p}<0.01$. C) Flow cytometry cell cycle assay. Cell cycle distribution was analyzed after transfection of MIG-6 siRNA into miR-589-5p inhibited HepG2 cells. ${ }^{\star} \mathrm{p}<0.05$ and ${ }^{\star *} \mathrm{p}<0.01$. 
other microRNA are reported to be aberrantly expressed in HCC and can act as biomarkers [16-21]. Although MiR-589-5p is a poorly studied microRNA in cancer, there is the following research; (1) Zhang et al. demonstrated that it inhibits MAP3K8 and suppresses CD90+ cancer stem cells in HCC [22]; (2) Long et al. found that it promotes the cancer stem cell characteristics and chemo-resistance of HCC cells by targeting multiple negative regulators of the STAT3 signaling pathway [23] and (3) Huang et al. reported that the expression of miR-589-5p is down-regulated in radiated laryngeal squamous carcinoma stem cells [24]. However, more biological effects of miR-589-5p need to be revealed.

Our work is supported by Long et al. who reported that miR-589-5p is elevated in HCC tissues [23]. Herein, we validate and extend their report. Our work indicated that miR-589-5p is highly expressed in HCC samples and cells. We identified that the level of miR-589-5p expression is associated with overall survival of HCC patients and that miR-589-5p could prove a novel HCC prognosis biomarker.

We then explored the roles that miR-589-5p plays in HCC. In vitro functional studies demonstrated that suppression of miR-589-5p significantly inhibited HCC cell growth, colony formation and cell cycle progression. These results indicate that miR-589-5p may function as an oncogene in HCC, because high microRNA expression by inhibition of tumor suppressor genes is generally an oncogene role [25]. We therefore searched the target genes of miR-589-5p. Bioinformatics tools revealed that MIG- 6 was a theoretical target of miR-589-5p and we confirmed this by qRT-PCR, Western blot and luciferase reporter assay.

MIG-6 is located on chromosome $1(1 \mathrm{p} 36.23)$ and was first reported in 1995 to have a role in cell cycle progression [26]. It is now reported to be a tumor-suppressor gene in many cancers [27], a negative regulator of the epidermal growth factor receptor signal [28] and an inhibitor of HCC epidermal growth factor receptor signaling and cell growth [29].Other research includes; (1) Li Z et al. reported MIG-6 is down-regulated in HCC and inhibits proliferation of HCC cells via P-ERK/Cyclin D1 pathway [30]: (2) Li H et al. found that miR-374a promotes HCC cell proliferation by targeting MIG-6 [31]; (3) Okada $\mathrm{H}$ et al. found that over-expression of miR-214 decreases the expression of MIG-6 and increases the levels of EGF-mediated p-EGFR and p-Met in Huh-7 cells [32] and (4) MIG-6 is reported to be involved in other tumors. For example, MIG-6 is regulated by MEK and affects EGF-induced migration in mutant NRAS melanoma [33] and MIG-6 up-regulation in endometrial carcinoma cells triggers anti-tumor effects and attenuates progesterone resistance [34]. Further, MIG-6 is also decreased in CCSPCreKras ${ }^{\text {G12D }}$ induced lung tumors and its down-regulation attenuates tumor cell apoptosis partly through activating the ErbB4 pathway [35].

Most importantly, herein we established that suppression of miR-589-5p increases MIG-6 expression in HCC cells and that miR-589-5p directly inhibits MIG-6 expres- sion. Moreover, we determined that miR-589-5p is highly expressed in HCC and that it can act a novel HCC prognosis biomarker. Finally, we confirmed that miR-589-5p has a carcinogenic role in HCC by targeting the MIG- 6 tumor suppressor gene, and our combined results suggest that miR-589-5p could be a novel target for HCC therapy.

Acknowledgements: This work was supported by the National Natural Science Foundation of China (No. 31571443 to QY), the Jilin Provincial Science and Technology Department (No. 20150101121JC to QY), and the Health and Family Planning Commission of Jilin Province (No. 2014 Z068 to QY).

\section{References}

[1] BOSCH FX, RIBES J, DIAZ M, CLERIES R. Primary liver cancer: worldwide incidence and trends. Gastroenterology 2004; 127: S5-S16.

[2] SIEGEL RL, MILLER KD, JEMAL A. Cancer Statistics, 2017. CA Cancer J Clin 2017; 67: 7-30. https://doi.org/10.3322/ caac. 21387

[3] EL-SERAG HB, RUDOLPH KL. Hepatocellular carcinoma: epidemiology and molecular carcinogenesis. Gastroenterology 2007; 132: 2557-2576. https://doi.org/10.1053/j.gastro.2007.04.061

[4] FORNER A, LLOVET JM, BRUIX J. Hepatocellular carcinoma. Lancet 2012; 379: 1245-1255. https://doi.org/10.1016/ s0140-6736(11)61347-0

[5] BRUIX J, GORES GJ, MAZZAFERRO V. Hepatocellular carcinoma: clinical frontiers and perspectives. Gut 2014; 63: 844-855. https://doi.org/10.1136/gutjnl-2013-306627

[6] SHUKLA GC, SINGH J, BARIK S. MicroRNAs: Processing, Maturation, Target Recognition and Regulatory Functions. Mol Cell Pharmacol 2011; 3: 83-92.

[7] BARTEL DP. MicroRNAs: genomics, biogenesis, mechanism, and function. Cell 2004; 116: 281-297.

[8] FARAZI TA, HOELL JI, MOROZOV P, TUSCHL T. MicroRNAs in human cancer Adv Exp Med Biol 2013; 774: 1-20. https://doi.org/10.1007/978-94-007-5590-1_1

[9] IORIO MV, CROCE CM. microRNA involvement in human cancer. Carcinogenesis 2012; 33: 1126-1133. https://doi. org/10.1093/carcin/bgs140

[10] AGARWAL V, BELL GW, NAM JW, BARTEL DP. Predicting effective microRNA target sites in mammalian mRNAs. Elife. 2015; 4. https://doi.org/10.7554/eLife.05005

[11] LI JH, LIU S, ZHOU H, QU LH, YANG JH. starBase v2.0: decoding miRNA-ceRNA, miRNA-ncRNA and proteinRNA interaction networks from large-scale CLIP-Seq data. Nucleic Acids Res 2014; 42: D92-97. https://doi.org/10.1093/ nar/gkt1248

[12] WONG N, WANG X. miRDB: an online resource for microRNA target prediction and functional annotations. Nucleic Acids Res 2015; 43: D146-152. https://doi.org/10.1093/ nar/gku1104

[13] BRUIX J, REIG M, SHERMAN M. Evidence-Based Diagnosis, Staging, and Treatment of Patients With Hepatocellular Carcinoma. Gastroenterology 2016; 150: 835-853. https:// doi.org/10.1053/j.gastro.2015.12.041 
[14] HANAHAN D, WEINBERG RA. Hallmarks of cancer: the next generation. Cell. 2011; 144: 646-674. https://doi. org/10.1016/j.cell.2011.02.013

[15] IORIO MV, CROCE CM. MicroRNA dysregulation in cancer: diagnostics, monitoring and therapeutics. A comprehensive review. EMBO Mol Med 2012; 4: 143-159. https:// doi.org/10.1002/emmm.201100209

[16] KOBERLE V, KRONENBERGER B, PLELI T, TROJAN J, IMELMANN E et al. Serum microRNA-1 and microRNA-122 are prognostic markers in patients with hepatocellular carcinoma. Eur J Cancer 2013; 49: 3442-3449. doi:10.1016/j.ejca.2013.06.002.

[17] LI J, WANG Y, YU W, CHEN J, LUO J. Expression of serum miR-221 in human hepatocellular carcinoma and its prognostic significance. Biochem Biophys Res Commun 2011; 406: 70-73. https://doi.org/10.1016/j.bbrc.2011.01.111

[18] BIHRER V, WAIDMANN O, FRIEDRICH-RUST M, FORESTIER N, SUSSER S et al. Serum microRNA-21 as marker for necroinflammation in hepatitis $\mathrm{C}$ patients with and without hepatocellular carcinoma. PLoS One 2011; 6: e26971. https://doi.org/10.1371/journal.pone.0026971

[19] YAMAMOTO Y, KOSAKA N, TANAKA M, KOIZUMI F, KANAI Y et al. MicroRNA-500 as a potential diagnostic marker for hepatocellular carcinoma. Biomarkers 2009; 14: 529-538. https://doi.org/10.3109/13547500903150771

[20] XIONG Y, FANG JH, YUN JP, YANG J, ZHANG Y et al. Effects of microRNA-29 on apoptosis, tumorigenicity, and prognosis of hepatocellular carcinoma. Hepatology 2010; 51: 836-845. https://doi.org/10.1002/hep.23380

[21] BOREL F, KONSTANTINOVA P, JANSEN PL. Diagnostic and therapeutic potential of miRNA signatures in patients with hepatocellular carcinoma. J Hepatology 2012; 56: 13711383. https://doi.org/10.1016/j.jhep.2011.11.026

[22] ZHANG X, JIANG P, SHUAI L, CHEN K, LI Z et al. miR589-5p inhibits MAP3K8 and suppresses CD90+ cancer stem cells in hepatocellular carcinoma. J Exp Clin Cancer Res 2016; 35: 176. https://doi.org/10.1186/s13046-016-04526

[23] LONG J, JIANG C, LIU B, DAI Q, HUA R et al. Maintenance of stemness by miR-589-5p in hepatocellular carcinoma cells promotes chemoresistance via STAT3 signaling. Cancer Lett 2018;423: 113-126. https://doi.org/10.1016/j.canlet.2017.11.031

[24] HUANG CX, ZHU Y, DUAN GL, YAO JF, LI ZY et al. Screening for MiRNAs related to laryngeal squamous carcinoma stem cell radiation. Asian Pacific journal of cancer prevention. Asian Pac J Cancer Prev 2013; 14: 4533-7453.
[25] VENTURA A, JACKS T. MicroRNAs and cancer: short RNAs go a long way. Cell 2009; 136: 586-591. https://doi. org/10.1016/j.cell.2009.02.005

[26] WICK M, BURGER C, FUNK M, MULLER R. Identification of a novel mitogen-inducible gene (mig-6): regulation during G1 progression and differentiation. Exp Cell Res 1995; 219: 527-535. https://doi.org/10.1006/excr.1995.1261

[27] ZHANG YW, STAAL B, SU Y, SWIATEK P, ZHAO P et al. Evidence that MIG-6 is a tumor-suppressor gene. Oncogene 2007; 26: 269-276. https://doi.org/10.1038/sj.onc.1209790

[28] HACKEL PO, GISHIZKY M, ULLRICH A. Mig-6 is a negative regulator of the epidermal growth factor receptor signal. Biol Chem 2001; 382: 1649-1662. https://doi.org/10.1515/ BC. 2001.200

[29] RESCHKE M, FERBY I, STEPNIAK E, SEITZER N, HORST $\mathrm{D}$ et al. Mitogen-inducible gene- 6 is a negative regulator of epidermal growth factor receptor signaling in hepatocytes and human hepatocellular carcinoma. Hepatology 2010; 51: 1383-1390. https://doi.org/10.1002/hep.23428

[30] LI Z, QU L, LUO W, TIAN Y, ZHAI H et al. Mig-6 is downregulated in HCC and inhibits the proliferation of HCC cells via the P-ERK/Cyclin D1 pathway. Exp Mol Pathol 2017; 102: 492-499. https://doi.org/10.1016/j.yexmp.2017.05.004

[31] LI H, CHEN H, WANG H, DONG Y, YIN M et al. MicroRNA-374a Promotes Hepatocellular Carcinoma Cell Proliferation by Targeting Mitogen-Inducible Gene-6 (MIG-6). Oncol Res 2017. https://doi.org/10.3727/0965040 17X15000784459799

[32] OKADA H, HONDA M, CAMPBELL JS, TAKEGOSHI K, SAKAI Y et al. Inhibition of microRNA-214 ameliorates hepatic fibrosis and tumor incidence in platelet-derived growth factor C transgenic mice. Cancer Sci 2015; 106: 1143-1152. https://doi.org/10.1111/cas.12730

[33] VU HL, ROSENBAUM S, CAPPARELLI C, PURWIN TJ, DAVIES MA et al. MIG6 Is MEK Regulated and Affects EGF-Induced Migration in Mutant NRAS Melanoma. J Invest Dermatol 2016; 136: 453-463. https://doi.org/10.1016/j. jid.2015.11.012

[34] XU W, ZHU S, ZHOU Y, JIN Y, DAI H et al. Upregulation of mitogen-inducible gene 6 triggers antitumor effect and attenuates progesterone resistance in endometrial carcinoma cells. Cancer Gene Ther 2015; 22: 536-541. https://doi. org/10.1038/cgt.2015.52

[35] LIU J, CHO SN, WU SP, JIN N, MOGHADDAM SJ et al. Mig-6 deficiency cooperates with oncogenic Kras to promote mouse lung tumorigenesis. Lung Cancer 2017; 112: 47-56. https://doi.org/10.1016/j.lungcan.2017.08.001 